\title{
DELAYED FDG-PET/CT IMAGES IN PATIENTS WITH BRAIN TUMORS - IMPACT ON VISUAL AND SEMIQUANTITATIVE ASSESSMENT
}

\author{
Pavel H. Bochev ${ }^{1}$, Aneliya Klisarova ${ }^{1}$, Ara Kaprelyan ${ }^{2}$, Borislav Chaushev ${ }^{1}$, Zhivka \\ Dancheva $^{1}$ \\ ${ }^{I}$ Department of Nuclear Medicine and Radiotherapy, "St. Marina" University \\ Hospital, Varna, Bulgaria \\ ${ }^{2}$ Department of Neurology, "St. Marina” University Hospital, Varna, Bulgaria
}

\section{ABSTRACT}

Background: Despite the extensive use of FDG-PET/ $\mathrm{CT}$ its role in brain tumor assessment remains controversial mostly because of the physiologically high brain uptake which easily obscures pathological processes. The wide availability of FDG, however, maintains the interest in FDG neuro-oncological applications.

Objective: to evaluate the use of a late registration at $180 \mathrm{~min}$ in patients with brain tumors, studied with FDGPET/CT based on visual and semiquantitative analysis.

Materials and methods: 38 patients with brain neoplasms and non-tumor structural lesions underwent a selective brain 18F-FDG PET/CT at two time points at 60 and 180 minutes after administration. Visual assessment was made by two readers with interobserver agreement calculation. Region ratio comparison with three different reference regions - the contralateral one, the white matter, and the cerebellum was used as a base for semiquantitative analysis.

Results: Visual analysis showed better delineation of malignant lesion on late registrations with higher inter/ intraobserver agreement as compared to the early images. Semiquantitative analysis demonstrated significant differences in early and late indices of metastases and gliomas, but failed in distinguishing gliomas from metastatic lesions and benign lesions.

Conclusion: Delayed brain images with FDG-PET/CT at $180 \mathrm{~min}$ after injection provide better tumor delineation, higher accuracy, lower interobserver variations. The use of semiquantitative indices, irrespective of the reference region used, is of limited value.

Key words: FDG PET/CT, brain tumors, dual time point image

\section{BACKGROUND}

Current utilization of FDG PET in oncology is extensive and well documented in different types of tumors, including brain neoplasms. [1]. Nevertheless, current utilization of FDG-PET in neuro-oncology remains controversial $[2,3,4,5,6]$. The main reason for the withdrawal from neuro-oncologic applications is the normal biodistribution of FDG, which is characterized by high activity of the brain structures, generally obscuring any pathological process of hypermetabolic type and providing a wide differential diagnosis for focal lesions of hypometabolic type. The physiological pattern of the brain glucose metabolism is characterized by high gray matter uptake, resulting in excessive activity in cortical structures, basal ganglia and thalamus and relatively lower activity in white matter. Modern PET/CT machines allow nearly simultaneous in line CT and PET acquisitions, generating three basic sets of images - PET, CT and combined, fused images. These technological advances improve the detectability and characterization of brain lesions on visual analysis and make semiquantitative analysis much more reliable due to the more precise targeting of the regions of interest (ROI). Combined PET/MRI scanners go even further, but are not widely available [7, 8]. A borderline compromise could be a software fusion of image sets, acquired with different PET and MRI machines [8]. Brain tumors, like any other malignant processes are characterized by specific level of FDG utilization. It is widely known that low grade gliomas have low FDG uptake, while high grade gliomas usually present with much higher uptake, this fact not being a strict rule. The gold standard for the assessment of the low grade gliomas, as far as it concerns nuclear medicine studies are labeled amino acids, while FDG PET is much more suitable for high grade gliomas assessment and pre-operative grading. In low grade gliomas, FDG PET is a good tool for the monitoring of malignant transformations of known tumors $[2,9]$. There are two main factors for brain lesions to be clearly visualized - high tumor uptake and/ or low brain uptake. It is known from the general PET/CT practice that hyperglycemia results in altered FDG biodistribution with lower brain uptake, which is a possible way to better the delineation of a brain tumor by glucose loading. This issue is discussed in the literature, but despite the promising 
results the method has not gained popularity, mainly because of its complicated preparation [10]. Another option for lowering the brain activity is to wait for the normal washout and register the images late after the administration of FDG. The aim is to achieve optimal wash-out from the normal structures without reaching a nadir in tumor activity. There is some literature addressing the utilization of dual phase $\mathrm{PET} / \mathrm{CT}$ of the brain with the delay interval ranging from 2 to 8 hours after FDG administration [11, 12, 13, 14]. We also adopted this technique selecting a baseline registration at $60^{\text {th }}$ minute and a three-hour delay time for the second registration.

The aim of our study was to evaluate the use of a late registration in patients with brain tumors, studied with FDG-PET/CT based on visual analysis and semiquantitative measurements.

\section{MATERIALS AND METHODS}

A total of thirty-eight (38) patients (21 females and 17 males) with brain tumors or suspected brain neoplasms were included in the study. Of them 14 had brain metastases, 11 - primary brain tumors, and 4 - non-tumor structural brain lesions. Nine (9) patients with medical history of operated brain tumors, but no recurrence on MRI were also included in the study (all underwent further clinical and imaging follow up for at least 18 months or until progression). In these 38 patients, overall 45 lesions were assessed visually and semiquantitatively. The group of primary brain tumors $(\mathrm{N}=11)$ included 2 patients with low grade astrocytomas (Grade II), 2 with anaplastic astrocytomas (Grade III), one with low grade oligodendriglioma (Grade II), 5 with meningiomas, and one patient with meningosarcoma. Nine (9) of the patients were studied preoperatively, two (2) of the patients with primary brain tumors were directed for PET/CT due to suspected recurrence. The group of metatstatic tumors $(\mathrm{N}=14)$ included 8 patients with lung cancer, 4 patients with breast cancer, one patient with ovarian cancer and one patient with metastases from unknown primary. The patients with non-tumor lesions were directed for PET/CT due to structural brain lesions with equivocal findings on CT - two with arachnoid cysts, one with cavernoma and one with suspected paraselar process, which was later excluded by MRI. The group of postoperative changes $(\mathrm{N}=9)$, although formally of nontumor origin were assessed separately (Table 1).
Table1. Grouping of patients and types of diagnostic verification.

\begin{tabular}{|l|c|l|}
\hline Groups & $\begin{array}{c}\text { N of } \\
\text { patients }\end{array}$ & Verification \\
\hline Glial & 5 & Operation \\
\hline Meningeal & 6 & Operation \\
\hline Metastases & 14 & CECT, MRI, operation \\
\hline $\begin{array}{l}\text { Operated } \\
\text { (postoperative changes) }\end{array}$ & 9 & MRI follow up \\
\hline Non- tumor lesions & 4 & MRI, clinical follow up \\
\hline
\end{tabular}

All PET/CT studies were performed with Philips Gemini TF PET/CT (2009) with 16slice CT and 3D TOF PET. FDG was injected manually through intravenous line in activity of $5.0 \mathrm{mCi}$ for sole brain scanning and $0.14 \mathrm{mCi} / \mathrm{kg}$ for patients, referred for whole body PET/CT. After FDG administration all patients rested in a dimmed quiet room for 60 minutes and were then scanned. Acquisition was performed with a standard manufacturer's protocol for brain scanning using LowDoseCT $120 \mathrm{Kev}, 50 \mathrm{mAs}$ and PET in a single $10 \mathrm{~min}$ frame with $256 \mathrm{mmFOV}, 2 \mathrm{~mm}$ pixel size. After the first registration, referred here as early registration, the patients spent additional 120 minutes (to up to $180 \mathrm{mrn}$ after FDG administration) in a shielded box and were then scanned again with the same protocol. The images were then assessed visually and semiquantitatively.

All patients signed informed consent. The final diagnosis was based on MRI and contrast enhanced CT (CECT, taken in consideration only for multiple brain metastases) and MRI follow-up. All primary brain tumors were verified histologically and so were four of the cases with a sole metastasis. The group of operated patients with no evidence of recurrence by the time of the FDG scan was followed by sequential MRI scans at least 18 months for all glial tumors and with routine cancer-specific follow up regimens for patients with operated brain metastases (3 of them died in a year from non-CNS progression, one still in remission).

Semiquantitative analysis: Region of interest (ROI) was placed over the hottest part of the lesion if hypermetabolic or over the lesion identified on CT, if not active. ROI was drawn as a spherical model in respect to the position in all three planes, but was not less than $1 \mathrm{~cm}^{3}$. Maximum pixel counts value was used for the calculations. Lesion ROI maximum counts/pixel were compared to contralateral ROI, ROI over white matter and ROI over cerebellum. ROI data was processed by comparison with the three references ROI's and three types of indices were derived as follows: lesion to contralateral $\mathrm{ROI}(\mathrm{L} / \mathrm{Cl})$, lesion to white matter $(\mathrm{L} / \mathrm{WM})$ and lesion to cerebellum $(\mathrm{L} / \mathrm{Crb})$. Indices were derived from early and late images. We 
calculated additional retention indices as late index to early index (L/E) by ROI. Early and late indices difference was tested for significance by paired Student's t-test (Excel). Differences of indices by groups were tested for significance by Student's t-test for unpaired data. The data was assessed additionally by receiver operating characteristic analysis (ROC) by plotting ROC curves and estimating the area under the curve as well as point-by-point testing of different cutoff values of the indices for optimal sensitivity and specificity trade-off. For the purposes of ROC analysis the patients were classified as a positive fraction (having tumor) and a negative fraction (tumor free), with meningiomas excluded from the analysis.

Visual assessment: The two image sets, obtained for every patient (late and early) were individually read by two nuclear medicine physicians and were scored in a three-step scale as follows: 0 - no evidence of tumor, 1 - equivocal, and 2 - positive for tumor. The datasets were anonymized. The readers were blind to any clinical data and whether they are reading early or late images.After the blind read, the two readers were made aware of the clinical data and the sequence of the scans and then subjectively assessed lesion delineation on early and late images and their contribution to the diagnosis. The decision was binary with 'yes' - clear contribution of late image and 'no' - no contribution.

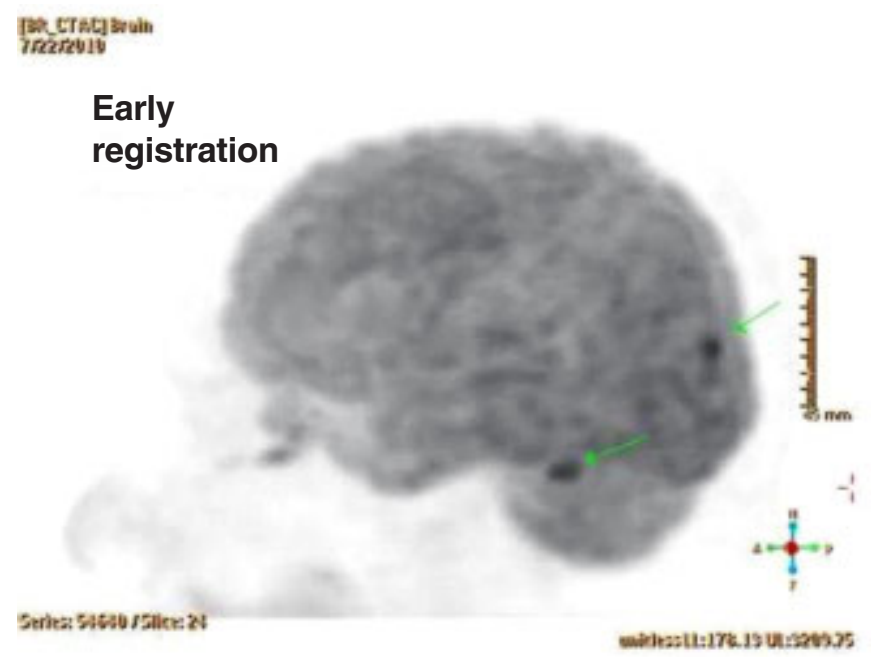

\section{RESULTS AND DISCUSSION}

Visual analysis consists of blind reading of both early and late images by two readers in order to asses interobserver agreement in reading for both image sets and subjective unblind assessment of the contribution of every image set to the final decision made by the two readers. Visual analysis showed lack of pathological hypermetabolism of all non-tumor lesions. All patients, surgically treated for brain tumors, but lacking evidence of recurrence, had a trend to strictly hypometabolic changes at, and in some cases around the surgery site. Patients with brain metastases presented with heterogeneous lesion activity, ranging from extremely hypermetabolic to clearly hypometabolic, including some equivocal findings with borderline activity and uncertain reading (Fig. 1). All patients with meningiomas showed no pathological activity, with a sole finding of slight (relative) hypometabolism of the lesion. One of the patients with gliomas was false-negative on PET, with an MRI showing a large brain tumor mass. The patient was histologically proven to have a low grade oligodendroglioma. The other glioma patients were identified by focal hypermetabolism, exceeding by far that of the grey matter.

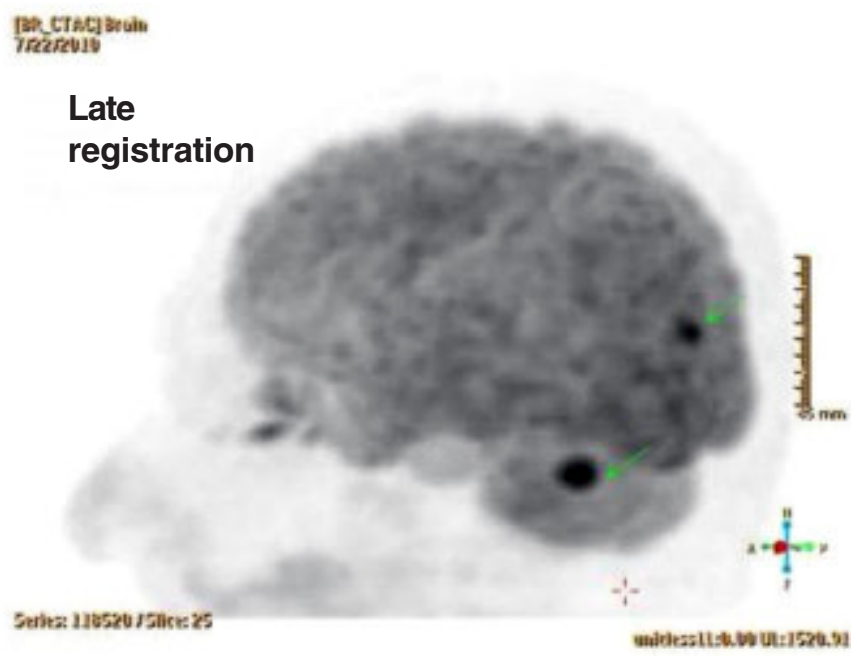

Fig. 1. A patient with two metastatic foci, faintly visualized at early image (left), but clearly delineated on late image (right).

Readers' score results for early and late images were tested for correlation (Pearson's R) and agreement (kappa) for both interobserver and intraobserver agreement. Statistical tests show a high interobserver correlation for late readings ( $\mathrm{r}=0.93)$, compared to early $(0.83)$. The measure of agreement (kappa) was highest for late interobserver comparison (0.87), lower for early sets comparison $(0.60)$ and inraobserver (early vs late) comparison (reader 1 - 0.60; reader 2 -0.79). After the blind reading, all data was revealed and the readers scored their subjective assessment of image quality and whether late images had solely influenced the final decision. In nearly all cases, late images were defined as more contrast and with a better delineation of the target lesion. Both readers score 15 resp. 14 cases in which late images are the only issue to justify the final decision.

Semiquantitative analysis: Based on the uptake 
values in the regions of interest, derived from both early and late registration, we calculated the mean and standard deviation for every ROI pair - early and late. The early and late indices calculated were compared by paired Student's t-test. Patients were divided in subgroups as follows - nontumor lesions (excluding postoperative changes) $(\mathrm{N}=4)$, postoperative changes (operated tumors with no further evidence of recurrence) $(\mathrm{N}=9)$, metastases - 21 in 13 patients, primary brain tumors $(\mathrm{N}=10)$, subdivided in gliomas $(\mathrm{N}=5)$ and meningiomas $(\mathrm{N}=5)$. Early and late indices (for all three ROIs) differ significantly only in the metastatic group $(\mathrm{p}<0.001)$. Higher difference significance is seen for contralateral ROI index and cerebellum index. Higher retention indices in the metastatic group are probably due to higher FDG retention in metastatic tumors than in normal tissue or gliomas. The glioma indices difference was significant only for lesion to contralateral ROI and borderline for cerebellum. However there is a trend toward significance $(\mathrm{p}=0.03)$ which is not noted in the non-tumor, meningeoma and postoperative group. Given the fact that metastases and gliomas are the most common object of differential diagnosis, we could assume that there is a significant difference in the target aggressive groups (gliomas, metastases) compared benign pathologies such as non-tumor lesions, meningeomas and postoperative changes.

Intergroup comparison of the indices by Student's ttest for unpaired data demonstrated a significant difference between the indices of non-tumor lesions and meningeomas on one side and metastases on the other $(\mathrm{p}<0.05)$ for all ROIs for both early and late images. Opposite to metastatic tumors, the gliomas indices did not differ significantly from those, derived for benign lesion and meningeomas $(p>0.05)$, neither on early nor on late images and retention indices, which is rather disappointing, because it is a common differential diagnosis issue.

ROC analysis: All indices data acquired were processed by ROC analysis in order to test the classifying value of all the indices derived and to define a discriminative cut-off value for positive and negative fraction. Automated ROC analysis was used by the means of SPSS software package. One patient's data was excluded due to diffuse changes in the contralateral hemisphere, which biased the semiquantitative data. The area under the curve differs significantly from the reference line for both early and late indices. Retention indices perform worse, especially for the white matter retention index whose under the curve area didn't differ significantly from the reference line which is in concordance with earlier studies [13].

\section{CONCLUSION}

Delayed brain images with FDG-PET/CT at $180 \mathrm{~min}$ after injection provide better tumor delineation, higher accuracy, lower interobserver variations and higher subjective acceptance of the images from the reader. Based on our results we consider their clinical use meaningful and recommendable. Delayed images do not provide a completely different picture from what is seen on initial one hour images but are more contrast and the number of equivocal readings is diminished. The use of semiquantitative late and early indices, irrespective of the reference region is of more limited value and should be used cautiously and with respect to the visual findings.

\section{REFERENCES:}

1. Di Chiro G, DeLaPaz RL, Brooks RA, Sokoloff L, Kornblith PL, Smith $\mathrm{BH}$, et al. Glucose utilization of cerebral gliomas measured by $[18 \mathrm{~F}]$ fluorodeoxyglucose and positron emission tomography. Neurology. 1982 Dec;32(12):1323-9. [PubMed]

2. Barker FG 2nd, Chang SM, Valk PE, Pounds TR, Prados MD. 18Fluorodeoxyglucose uptake and survival of patients with suspected recurrent malignant glioma. Cancer. 1997 Jan 1;79(1):115-26. [PubMed]

3. De Witte O, Levivier M, Violon P, Salmon I, Damhaut P, Wikler D Jr, et al. Prognostic value of positron emission tomography with [18F] fluoro-2-Dglucose in the low-grade glioma. $J$ Neurosurg. 1996 Sep;39(3):470-477.

\section{[PubMed]}

4. Di Chiro G, Oldfield E, Wright DC, De Michele D, Katz DA, Patronas NJ, et al. Cerebral necrosis after radiotherapy and/or intraarterial chemotherapy for brain tumors: PET and neuropathologic studies. AJR Am J Roentgenol. 1988 Jan;150(1):189-197. [PubMed]

5. Patronas NJ, Di Chiro G, Brooks RA, DeLaPaz RL, Kornblith PL, Smith $\mathrm{BH}$, et al. Work in progress: [18F] fluorodeoxyglucose and positron emission tomography in the evaluation of radiation necrosis of the brain. Radiology. 1982 Sep;144(4):885-889. [PubMed]

6. Ricci PE, Karis JP, Heiserman JE, Fram EK, Bice AN, Drayer BP.
Differentiating Recurrent Tumor from Radiation Necrosis: Time for Reevaluation of Positron Emission Tomography? AJNR Am J Neuroradiol. 1998 Mar;19(3):407-413. [PubMed]

7. Boss A, Bisdas S, Kolb A, Hofmann M, Ernemann U, Claussen $\mathrm{CD}$, et al. Hybrid PET/MRI of intracranial masses: initial experiences and comparison to PET/CT. J Nucl Med. 2010 Aug;51(8):1198-205. [PubMed] [CrossRef]

8. Townsend DW. Dual-modality imaging: combining anatomy and function. J Nucl Med. 2008 Jun; 49(6): 938-955. [PubMed] [CrossRef]

9. Padoma MV, Said S, Jacobs M, Hwang DR, Dunigan K, Satter M, et al. Prediction of pathology and survival 
by FDG PET in gliomas. $J$ Neurooncol. 2003 Sep;64(3):227-37. [PubMed]

10. Ishizu K, Nishizawa S, Yonekura Y, Sadato N, Magata Y, Tamaki N, et al. Effects of hyperglycemia on FDG uptake in human brain and glioma. $J$ Nucl Med. 1994 Jul;35(7):1104-1109. [PubMed]

11. Spence AM, Muzi M, Mankoff DA, O'Sullivan SF, Link JM, Lewellen TK, et al. 18F-FDG PET of gliomas at delayed intervals: improved distinction between tumor and normal gray matter. J Nucl Med. 2004 Oct;45(10):1653-9. [PubMed]

12. Horky LL, Hsiao EM, Weiss SE, Drappatz J, Gerbaudo VH. Dual phase FDG-PET imaging of brain metastases provides superior assessment of recurrence versus post-treatment necrosis. J Neurooncol. 2011 May; 103(1):137-46. [PubMed] [CrossRef]

13. Kim DW, Jung SA, Kim CG, Park SA. The efficacy of dual time point F-
18 FDG PET imaging for grading of brain tumors. Clin Nucl Med. 2010 Jun; 35(6):400-3. [PubMed] [CrossRef]

14. Prieto E, Marth-Climent JM, Domnnguez-Prado I, Garrastachu P, Diez-Valle R, Tejada S, et al. Voxelbased analysis of dual-time-point 18FFDG PET images for brain tumor identification and delineation. $\mathrm{J} \mathrm{Nucl}$ Med. 2011 Jun;52(6):865-72. [PubMed] [CrossRef]

Address for correspondence:

Dr Pavel H. Bochev

Department of Nuclear Medicine and radiotherapy, "St. Marina” University Hospital, Varna, Bulgaria

1 "Hristo Smirnenski” Str., Varna, Bulgaria

Tel: +35952978 331, Mobile:+359 887290457

E-mail: pbochev@yahoo.com; 